\title{
\begin{tabular}{l|l} 
Mitraries & DSpace@MIT
\end{tabular}
}

\author{
MIT Open Access Articles
}

\section{PHOTOMETRICALLY DERIVED MASSES AND RADII OF THE PLANET AND STAR IN THE TrES-2 SYSTEM}

The MIT Faculty has made this article openly available. Please share how this access benefits you. Your story matters.

Citation: Barclay, Thomas, Daniel Huber, Jason F. Rowe, Jonathan J. Fortney, Caroline V. Morley, Elisa V. Quintana, Daniel C. Fabrycky, et al. "PHOTOMETRICALLY DERIVED MASSES AND RADII OF THE PLANET AND STAR IN THE TrES-2 SYSTEM.” The Astrophysical Journal 761, no. 1 (November 21, 2012): 53. (C) 2012 The American Astronomical Society

As Published: http://dx.doi.org/10.1088/0004-637x/761/1/53

Publisher: IOP Publishing

Persistent URL: http://hdl.handle.net/1721.1/95638

Version: Final published version: final published article, as it appeared in a journal, conference proceedings, or other formally published context

Terms of Use: Article is made available in accordance with the publisher's policy and may be subject to US copyright law. Please refer to the publisher's site for terms of use. 


\title{
PHOTOMETRICALLY DERIVED MASSES AND RADII OF THE PLANET AND STAR IN THE TrES-2 SYSTEM
}

\author{
Thomas Barclay $Y^{1,2}$, Daniel Huber ${ }^{1,11}$, Jason F. Rowe ${ }^{1,3}$, Jonathan J. Fortney ${ }^{4}$, Caroline V. Morley ${ }^{4}$, \\ Elisa V. Quintana ${ }^{1,3}$, Daniel C. Fabrycky ${ }^{4,12}$, Geert Barentsen ${ }^{5}$, Steven Bloemen ${ }^{6}$, Jessie L. Christiansen ${ }^{1,3}$, \\ Brice-Olivier Demory ${ }^{7}$, Benjamin J. Fulton ${ }^{8}$, Jon M. Jenkins ${ }^{1,3}$, Fergal Mullally $^{1,3}$, Darin Ragozzine ${ }^{9}$, \\ Shaun E. Seader ${ }^{1,3}$, Avi Shrorer ${ }^{8,10}$, Peter Tenenbaum ${ }^{1,3}$, and Susan E. Thompson ${ }^{1,3}$ \\ ${ }^{1}$ NASA Ames Research Center, M/S 244-30, Moffett Field, CA 94035, USA \\ 2 Bay Area Environmental Research Institute, Inc., 560 Third Street West, Sonoma, CA 95476, USA \\ ${ }^{3}$ SETI Institute, 189 Bernardo Avenue, Suite 100, Mountain View, CA 94043, USA \\ ${ }^{4}$ Department of Astronomy and Astrophysics, University of California, Santa Cruz, CA 95064, USA \\ 5 Armagh Observatory, College Hill, Armagh BT61 9DG, UK \\ ${ }^{6}$ Instituut voor Sterrenkunde, Katholieke Universiteit Leuven, Celestijnenlaan 200 D, B-3001 Leuven, Belgium \\ ${ }^{7}$ Department of Earth, Atmospheric and Planetary Sciences, Massachusetts Institute of Technology, \\ 77 Massachusetts Avenue, Cambridge, MA 02139, USA \\ ${ }^{8}$ Las Cumbres Observatory Global Telescope Network, 6740 Cortona Drive, Suite 102, Santa Barbara, CA 93117, USA \\ ${ }^{9}$ Harvard-Smithsonian Center for Astrophysics, 60 Garden Street, Cambridge, MA 02138, USA \\ ${ }^{10}$ Department of Physics, Broida Hall, University of California, Santa Barbara, CA 93106, USA \\ Received 2012 August 16; accepted 2012 October 15; published 2012 November 21
}

\begin{abstract}
We measure the mass and radius of the star and planet in the TrES-2 system using 2.7 years of observations by the Kepler spacecraft. The light curve shows evidence for ellipsoidal variations and Doppler beaming on a period consistent with the orbital period of the planet with amplitudes of $2.79_{-0.62}^{+0.44}$ and $3.44_{-0.37}^{+0.32}$ parts per million (ppm), respectively, and a difference between the dayside and the nightside planetary flux of $3.41_{-0.82}^{+0.55} \mathrm{ppm}$. We present an asteroseismic analysis of solar-like oscillations on TrES-2A which we use to calculate the stellar mass of $0.94 \pm 0.05 M_{\odot}$ and radius of $0.95 \pm 0.02 R_{\odot}$. Using these stellar parameters, a transit model fit and the phase-curve variations, we determine the planetary radius of $1.162_{-0.024}^{+0.020} R_{\text {Jup }}$ and derive a mass for TrES-2b from the photometry of $1.44 \pm 0.21 M_{\text {Jup }}$. The ratio of the ellipsoidal variation to the Doppler beaming amplitudes agrees to better than $2 \sigma$ with theoretical predications, while our measured planet mass and radius agree within $2 \sigma$ of previously published values based on spectroscopic radial velocity measurements. We measure a geometric albedo of $0.0136_{-0.0033}^{+0.0022}$ and an occultation (secondary eclipse) depth of $6.5_{-1.8}^{+1.7} \mathrm{ppm}$ which we combined with the day/night planetary flux ratio to model the atmosphere of TrES-2b. We find that an atmosphere model that contains a temperature inversion is strongly preferred. We hypothesize that the Kepler bandpass probes a significantly greater atmospheric depth on the night side relative to the day side.
\end{abstract}

Key words: planets and satellites: individual (TrES-2b) - stars: individual (TrES-2A) - techniques: photometric

Online-only material: color figures

\section{INTRODUCTION}

The two most productive methods for discovering exoplanets have been surveys hunting for periodic changes in radial velocity motion seen in stellar spectra (e.g., Vogt et al. 2000; Mayor et al. 2009) and photometric searches for planetary transits (e.g., Bakos et al. 2002; Alonso et al. 2004; McCullough et al. 2005; Pollacco et al. 2006). From the radial velocity method the planet's minimum mass $(M \sin i)$ can be derived while the transit method allows for the measurement of the planetary radius and the orbital inclination. Neither technique can give the planet's density when used alone and knowledge of the stellar radius and mass is required for the radial velocity and transit methods, respectively.

Several dozen transiting planets have a measured density from follow-up radial velocity observations (both http://exoplanet.eu (Schneider et al. 2011) and http://exoplanets.org (Wright et al. 2011) provide up-to-date lists of planetary characteristics), but this requires a significant amount of telescope time and the use

\footnotetext{
${ }^{11}$ NASA Postdoctoral Program Fellow.

${ }^{12}$ Hubble Fellow.
}

of high-precision spectrographs. Until recently, no planet has had a mass derived from photometry alone. The first planets with masses measured from photometry were found through observations of transit timing variations (e.g., Lissauer et al. 2011; Ford et al. 2012a, 2012b; Steffen et al. 2012a; Fabrycky et al. 2012). However, this technique is limited to systems with multiple dynamically interacting planets.

The analysis of the light curves of transiting exoplanet host stars using space-based photometers has enabled the measurement of ellipsoidal variations (a recent review is given in Mazeh 2008) and Doppler beaming caused by transiting planets from which the planetary mass can be determined (Hills \& Dale 1974; Maxted et al. 2000; Loeb \& Gaudi 2003; Zucker et al. 2007). Ellipsoidal variations are periodic flux variations resulting from an orbiting body raising a tide on the host star. The star is therefore non-spherical and varies in brightness as a function of the visible surface area. The dominant period of this signal will be half the orbital period of the planet. Doppler beaming is a combination of a bolometric and a bandpassdependent effect. As a star moves due to the gravitational pull of a companion, the angular distribution of stellar flux will be beamed in the direction of the star's velocity vector. This effect 
is bolometric and results in an observed periodic brightness change proportional to the star's radial velocity. The bandpassdependent effect is a periodic redshift/blueshift in the spectral energy distribution of the star which results in the measured brightness of the star changing as the flux falling within the bandpass changes. The magnitude of this effect depends not only on the radial velocity of the star but also on the stellar spectrum and the observational bandpass.

The number of stars for which either planetary-induced ellipsoidal variation or Doppler beaming has been observed can be counted on one hand. Mazeh \& Faigler (2010) observed ellipsoidal variations and Doppler beaming in the phase curve (the complete flux time series of the star folded on the planetary orbital period) of CoRoT-3, the host to a $22 M_{\text {Jup }}$ planet/brown dwarf. More recently, Kepler observations of HAT-P-7 (Welsh et al. 2010; Mislis et al. 2012) and KOI-13.01 (Shporer et al. 2011; Mislis \& Hodgkin 2012; Mazeh et al. 2012) have been used to derive planet masses photometrically. Using the whole phase curve has also been shown to be a promising method for confirming the planetary nature of sources without the need for follow-up observations (Quintana et al. 2012).

Light from the planet itself also contributes to the out-oftransit phase-curve profile of host stars. The optical light from a cool planet is mainly owing to scattered starlight from the host star but hotter planets, such as the population of hot Jupiters, also contribute significant thermal emission at both infrared and optical wavelengths. From the ratio of a planet's flux (the combination of thermal emission and scattered light) to the stellar flux measured at full phase, the geometric albedo can be computed. The optical planet-to-star flux ratio has been measured for a small number of systems (e.g., Snellen et al. 2009; Welsh et al. 2010; Demory et al. 2011; Fortney et al. 2011; Désert et al. 2011a, 2011b) and robust upper limits have been placed on others (Rowe et al. 2008; Kipping \& Spiegel 2011). Combining a measurement of an occultation (secondary eclipse) depth and the star-to-planet flux ratio can help determine the planet's nightside and dayside equilibrium temperatures (Harrington et al. 2006), atmospheric composition (Rowe et al. 2006), heat redistribution (Showman et al. 2009), and even changing exoplanet weather patterns.

The study of exoplanets is often limited by the difficulty in determining stellar masses and radii from spectroscopy alone due to the strong degeneracies between effective temperature, surface gravity, and metallicity (e.g., Torres et al. 2012). Uncertainties on a spectroscopically derived radius can be at the level of 50\% (Basu et al. 2012). Asteroseismic observations of solar-like oscillations can be used to reduce these uncertainties to less than 5\% (e.g., Stello et al. 2009b; Chaplin et al. 2011; Carter et al. 2012).

In this work, we analyze the photometric time-series observations of the planet host star TrES-2A ${ }^{13}$ (listed in the Kepler Input Catalog with identification number 11446443) made using the Kepler spacecraft. We simultaneously fit transit, occultation, and phase-curve effects to determine the radius of TrES-2b ${ }^{14}$ and derive the mass of the planet by measuring the amplitude of Doppler beaming and ellipsoidal variations. TrES-2 is an ideal target for phase-curve analysis because (1) the star is relatively bright ( $V=11.4)$, (2) it has been observed by Kepler

\footnotetext{
13 In an attempt to avoid confusion, we use the designation TrES-2 to refer to the star system, TrES-2A to refer to the star, and TReS-2b to refer to the planet.

14 This source has the Kepler Object of Interest designation KOI-1.01.
}

in short-cadence mode since the beginning of the mission, ${ }^{15}$ (3) we detect solar-like oscillations on the star, (4) we see very little stellar activity, (5) the orbit of TrES-2b is very close to circular (Husnoo et al. 2012), (6) no variations in the transit times of TrES-2b are detected (Steffen et al. 2012b), and (7) a detection of light from the planet has previously been claimed (indeed, this planet has previously been reported to be the darkest known exoplanet; Kipping \& Spiegel 2011) and a robust upper limit on a secondary eclipse depth has previously been reported (Coughlin \& López-Morales 2012).

\section{OBSERVATIONS OF TrES-2}

The Kepler spacecraft was launched in 2009 with the goal of discovering transiting exoplanets (Borucki et al. 2010; Koch et al. 2010). The planet TrES-2b is one of only three to be discovered before the launch of Kepler (O'Donovan et al. 2006). These three previously known exoplanets have been observed in short-cadence mode (Gilliland et al. 2010) since the start of the mission. We use the Quarters 0-11 short-cadence observations (where the short-cadence sampling rate is $58.85 \mathrm{~s}$ ) of TrES-2 which span a total of 978 days. No data are used for Q4 and Q8 owing to star falling on a failed CCD during these quarters. We used simple aperture photometry data (shown in the top panel in Figure 1) as opposed to time series which have undergone pre-search data conditioning (PDC) as currently available shortcadence PDC data can distort astrophysical signals on timescales comparable to the orbital period of TrES-2b (Stumpe et al. 2012; Smith et al. 2012). We removed instrumental signals by fitting cotrending basis vectors to the time series of each quarter individually using the PYKE Kepler community software. ${ }^{16}$ Details of the cotrending basis vectors are given in the Kepler Data Processing Handbook (Christiansen et al. 2012) and their use is described in Barclay et al. (2012) and Kinemuchi et al. (2012). The cotrending basis vectors are only calculated for Kepler long-cadence data (29.4 minute sampling), so we interpolated onto the short-cadence time stamps using cubic splines. The light curve after the application of the cotrending basis vectors is shown in the lower panel of Figure 1.

In order to remove quarter-to-quarter discontinuities, we normalized each quarter to the median. Doing this is appropriate because contamination from background sources is very low for TrES-2 (typically less than $2 \%^{17}$ ). When analyzing transit photometry, it is common for data to be high-pass filtered in order to remove intrinsic stellar variability. However, we found this unnecessary because TrES-2A is intrinsically fairly quiet on timescale of days - there is little discernible variability owing to starspots. Filtering has the potential to damp the signals we are interested in and so being able to avoid filtering is highly preferable.

\section{THE STELLAR PARAMETERS OF TrES-2A}

The stellar properties of TrES-2A have so far mainly been studied using high-resolution spectroscopy. Sozzetti et al. (2007) combined constraints on the stellar density from the transit fit by Holman et al. (2007) to derive surface gravity, $\log g=4.44 \pm$ 0.02 , effective temperature, $T_{\text {eff }}=5850 \pm 50 \mathrm{~K}$, and metallicity, $[\mathrm{Fe} / \mathrm{H}]=-0.15 \pm 0.1$, while Ammler-von Eiff et al. (2009)

\footnotetext{
15 In short-cadence mode the sampling is approximately 1 minute as opposed to the standard Kepler sampling of $\sim 30$ minutes (Gilliland et al. 2010).

16 PyKE is available from http://keplergo.arc.nasa.gov/PyKE.shtml.

17 Contamination values for each source can be found on the data search Web site hosted by MAST.
} 


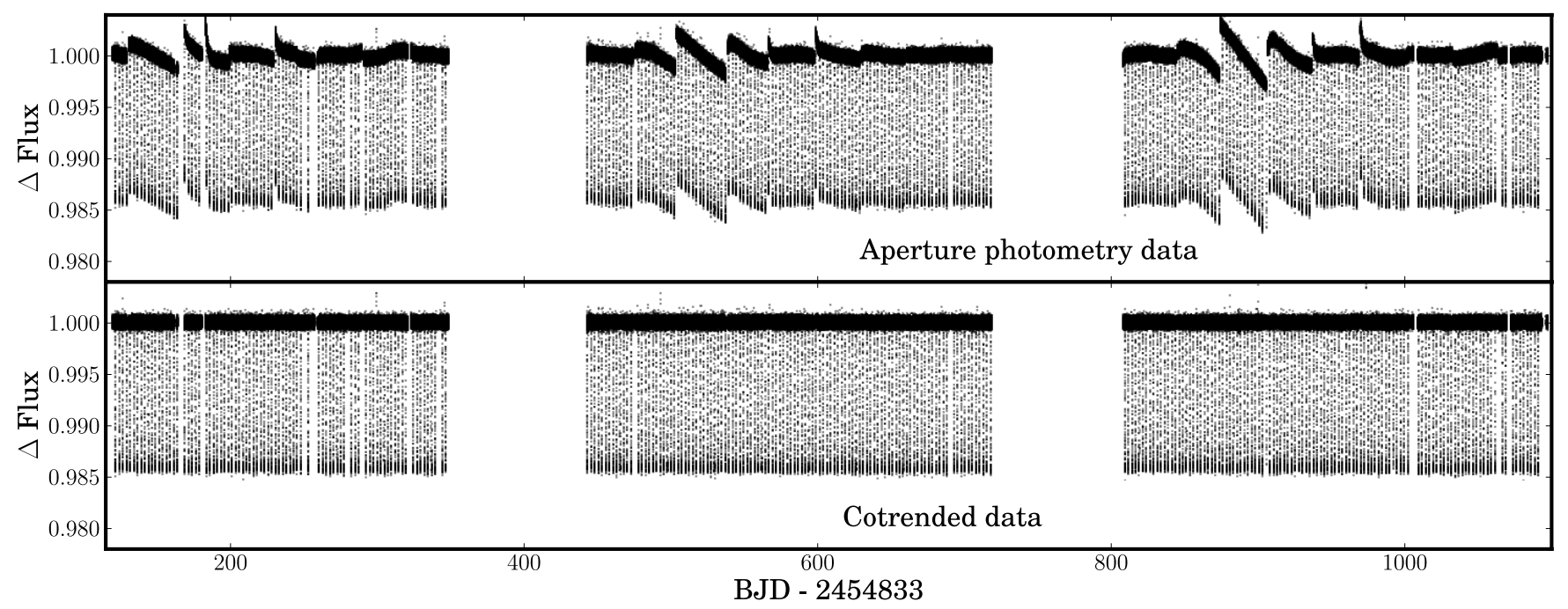

Figure 1. Simple aperture photometry data (called SAP_FLUX in the Kepler FITS files) is shown in the upper panel for available quarters up to Quarter 11. The data from which a combination of linear trends has been removed using the cotrending basis vectors are shown in the lower panel. Data have been normalized to the median for each quarter individually. The gaps in data are owing to the star falling onto Module 3 of the spacecraft photometer during Quarters 4 and 8. Module 3 failed during Quarter 4 . The regular pattern of transits can clearly be seen. The short sections of data where transits appear to be "missed" are because no data were collected during these times owing to events such as telescope Earth points and safe modes.

derived $\log g=4.3 \pm 0.1, T_{\text {eff }}=5795 \pm 73 \mathrm{~K}$, and $[\mathrm{Fe} / \mathrm{H}]=$ $0.06 \pm 0.08$ using spectroscopy only. Additionally, Southworth (2009) used $T_{\text {eff }}$ and $[\mathrm{Fe} / \mathrm{H}]$ from Sozzetti et al. (2007) together with empirical mass-radius relationship constraints from radial velocity measurements and the transit light curve to determine a mass and radius of $M / M_{\odot}=0.96 \pm 0.08$ and $R / R_{\odot}=$ $0.98 \pm 0.05$, respectively.

TrES-2A was among the first exoplanet host stars in the Kepler field to be analyzed using asteroseismology. The initial analysis by Christensen-Dalsgaard et al. (2010), based on the first 40 days of data, yielded a tentative detection of equally spaced peaks which was found to be in rough agreement with the properties derived by Sozzetti et al. (2007). To improve the stellar properties, we have analyzed all available shortcadence data up to Q11 using the method described by Huber et al. (2009). In summary, the method employs a frequencyresolved autocorrelation to locate the excess power due to solar-like oscillations. After correcting the power spectrum for contributions due to granulation, the large-frequency separation (the average spacing between oscillation modes of the same spherical degree and consecutive radial order) is calculated by fitting a Gaussian function to the highest peak of the power spectrum autocorrelation centered on the frequency of maximum power. We note that the method by Huber et al. (2009) has been thoroughly tested against independent methods (Hekker et al. 2011; Verner et al. 2011) and has been extensively applied for the analysis of single stars and large ensembles of stars observed with Kepler (see, e.g., Huber et al. 2011; Howell et al. 2012; Silva Aguirre et al. 2012).

Our analysis yielded a clear detection of excess power near $3400 \mu \mathrm{Hz}$, consistent with solar-like oscillations. Figure 2(a) displays the power spectrum centered around the power excess. Note that we have checked the frequency range for known artifacts in Kepler short-cadence data (see, e.g., Gilliland et al. 2010) and found no corresponding peaks in our data. The characteristic regular spacing indicative of $p$-mode oscillations is visible. We find $\Delta v=141.0 \pm 1.4 \mu \mathrm{Hz}$, with uncertainties estimated using Monte Carlo simulations. Figure 2(c) shows the power spectrum shown in Figure 2(a) after folding it on a frequency of $141 \mu \mathrm{Hz}$
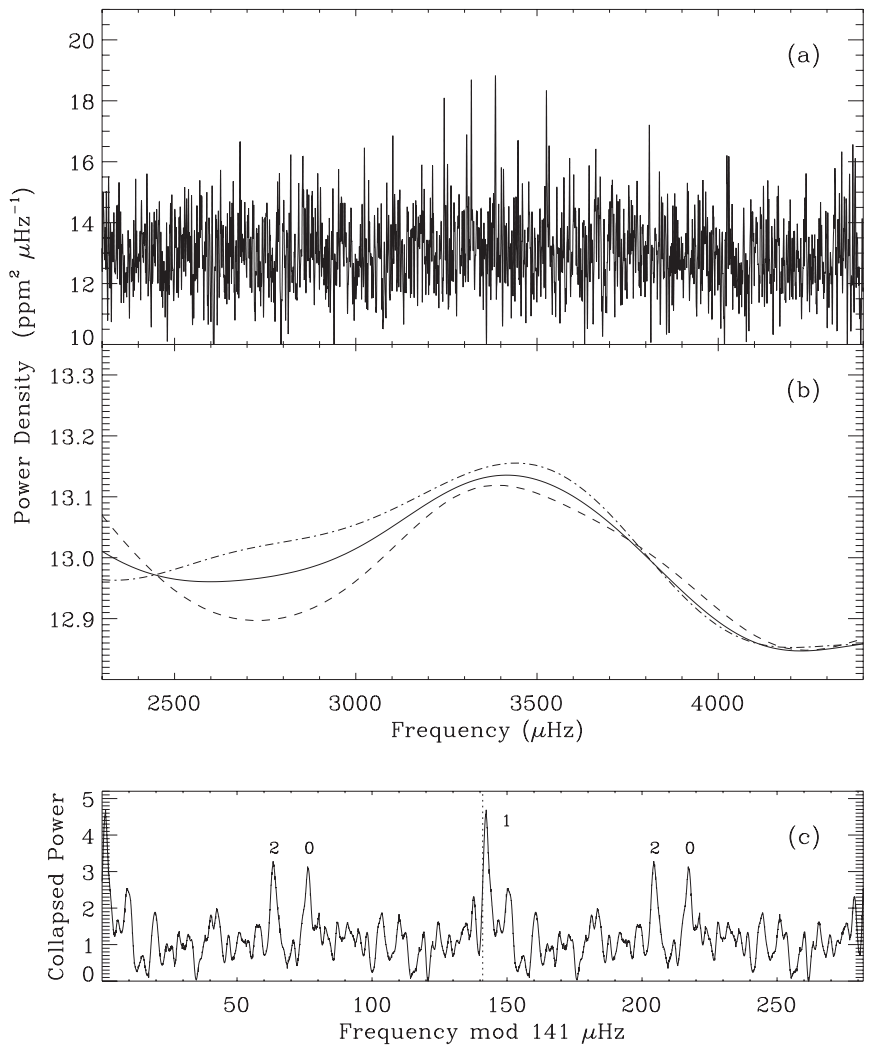

Figure 2. (a) The power spectrum of the Kepler Q0-Q11 time series of TrES-2A smoothed with a Gaussian filter of FWHM of $0.5 \mu \mathrm{Hz}$ and centered on the power excess due to solar-like oscillations. (b) Power spectrum shown in panel (a) but heavily smoothed with a FWHM of $4 \Delta v$ (solid line), compared to an equally smoothed power spectrum calculated using the first half (dashed line) and second half (dash-dotted line) of the data set. (c) The power spectrum shown in panel (a) folded on large-frequency separation between 2900 and $3800 \mu \mathrm{Hz}$ and binned. Plotted is the sum of the power in each bin. Numbers in the plot denote the identification of the spherical degree of each of the three visible ridges. The folded plot is shown twice for clarity. The dotted line marks the point where the plot repeats itself. 
Table 1

Stellar Properties of TrES-2

\begin{tabular}{lcc}
\hline \hline Property & Value & Reference \\
\hline$T_{\text {eff }}(\mathrm{K})$ & $5850 \pm 50$ & Sozzetti et al. (2007) \\
{$[\mathrm{Fe} / \mathrm{H}](\mathrm{dex})$} & $-0.15 \pm 0.1$ & Sozzetti et al. (2007) \\
$\rho\left(\mathrm{g} \mathrm{cm}^{-3}\right)$ & $1.53 \pm 0.03$ & This work \\
$M\left(M_{\odot}\right)$ & $0.94 \pm 0.05$ & This work \\
$R\left(R_{\odot}\right)$ & $0.95 \pm 0.02$ & This work \\
$\log g(\mathrm{dex})$ & $4.45 \pm 0.01$ & This work \\
Age $(\mathrm{Gyr})$ & $5.8 \pm 2.2$ & This work \\
\hline
\end{tabular}

and summing up all power between 2900 and $3800 \mu \mathrm{Hz}$, which roughly corresponds to the frequency range where oscillation modes are visible. There are three peaks in the folded power spectrum which we identify as oscillation modes of spherical degree $\ell=0,1$, and 2 . The measured small-frequency separation, the amount by which $\ell=2$ modes are offset from $\ell=0$ modes, from the folded spectrum is $\delta v_{02}=12.7 \mu \mathrm{Hz}$, which is fully consistent with a Sun-like star (e.g., Christensen-Dalsgaard 1988; White et al. 2011). The $\mathrm{S} / \mathrm{N}$ is not high enough to precisely constrain the frequency of maximum power $v_{\max }$ and hence it has not been used in the remainder of this analysis.

To test the significance of the detection, we first divided the data in two parts and calculated the power spectrum of each data set. The result is shown in Figure 2(b), which shows the power spectra after heavily smoothing them with a Gaussian filter with a $\mathrm{FWHM}=4 \Delta v$ to suppress the stochastic nature of the signal (see Kjeldsen et al. 2008). The excess power at $\sim 3400 \mu \mathrm{Hz}$ is visible in both data sets, confirming that the excess power in the combined data set is present in independent parts of the data. We furthermore performed simulations by generating time series with white noise corresponding to the time-domain scatter of the original data and by randomly shuffling the original data with replacement. For each simulated data set we performed the same analysis as for the original data. The results showed that the $l=1$ peak in the collapsed power spectrum (which primarily constrains the large separation) is detected at a level of $4.2 \sigma$, corresponding to a $>99.99 \%$ probability that the peak is not due to random noise. These tests, combined with the fact that the large-frequency separation, small-frequency separation, and location of the power excess are fully consistent for a solar-type star, give us confidence that the detected asteroseismic signal is robust.

The large separation can be shown to be directly related to the mean density of the star (Ulrich 1986):

$$
\frac{\Delta v}{\Delta v_{\odot}} \approx \sqrt{\frac{\rho_{*}}{\rho_{\odot}}} .
$$

Equation (1) has been tested both theoretically (e.g., Stello et al. 2009a; White et al. 2011) and empirically (e.g., Miglio 2012; Huber et al. 2012). Using our measured value above we arrive at a mean stellar density of $\rho_{\star}=1.53 \pm 0.03 \mathrm{~g} \mathrm{~cm}^{-3}$ for TrES-2. To estimate a full set of stellar parameters, we adopt $T_{\text {eff }}$ and $[\mathrm{Fe} / \mathrm{H}]$ from Sozzetti et al. (2007) and compare our constraints with a dense grid of quadratically interpolated BaSTI stellar evolutionary tracks (Pietrinferni et al. 2004). We start by identifying the maximum likelihood model assuming Gaussian likelihood functions for $\rho_{\star}, T_{\text {eff }}$, and $[\mathrm{Fe} / \mathrm{H}]$ (e.g., Basu et al. 2010; Kallinger et al. 2010). This procedure is then repeated $10^{5}$ times using values for $\rho_{\star}, T_{\text {eff }}$, and $[\mathrm{Fe} / \mathrm{H}]$ drawn from random distributions with standard deviations corresponding to the measurement uncertainty in each parameter. The final modeled

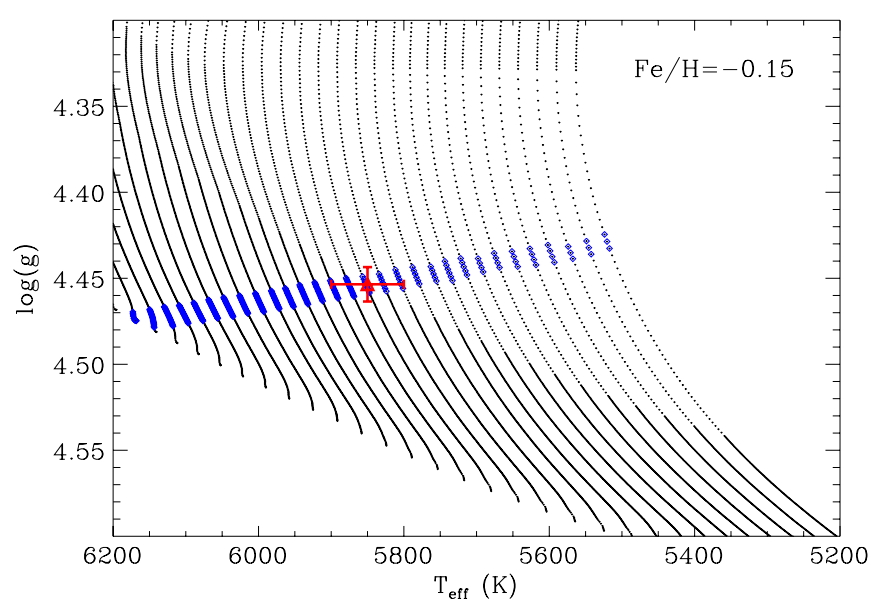

Figure 3. Surface gravity vs. effective temperature for quadratically interpolated BaSTI evolutionary tracks with masses from 0.8 to $1.1 M_{\odot}$ in steps of $0.01 M_{\odot}$ for the metallicity given by Sozzetti et al. (2007). Blue lines mark all models that satisfy the $1 \sigma$ constraint on the stellar density from asteroseismology. The red triangle shows derived best-fitting surface gravity with the effective temperature found by Sozzetti et al. (2007).

(A color version of this figure is available in the online journal.)

parameters and uncertainties are calculated as the median and 84.1 and 15.9 percentile of the resulting distributions. These stellar parameters are listed in Table 1.

Figure 3 shows evolutionary tracks in a $\log g-T_{\text {eff }}$ plane for the metallicity found by Sozzetti et al. (2007), illustrating the density constraint from the solar-like oscillations in the blue and showing our best-fitting model as a red triangle with uncertainties. We note that the uncertainties on the stellar properties stated in Table 1 do not include contributions from uncertainties due to different model grids, which have been shown to be on the order of $2 \%$ in radius and $5 \%$ in mass (e.g., Howell et al. 2012). We note that although some systematics in the stellar parameters can be expected because of the adopted model grid, previous analyses using a variety of stellar evolutionary models have shown that such systematics are generally not larger than the relative uncertainties quoted here (e.g., Howell et al. 2012).

The stellar properties from our analysis are in reasonably good agreement with the values from Sozzetti et al. (2007) and Southworth (2009). Our analysis presented here gives a direct measurement of the stellar density, resulting in refined stellar parameters which are independent of the transit modeling. We note that a more detailed asteroseismic analysis using individual frequencies, including constraints from the smallfrequency separations, can be expected to further improve the stellar parameters (in particular the age) of TrES-2A.

\section{FLUX TIME SERIES FITTING OF TrES-2B}

We fit a Mandel \& Agol (2002) transit model to the light curve of TrES-2 using a four-parameter nonlinear limb-darkening law with coefficients interpolated from Claret \& Bloemen (2011) using ATLAS models, where the interpolation is trilinear in $T_{\mathrm{eff}}$, $[\mathrm{Fe} / \mathrm{H}]$ and $\log g$. The four limb-darkening coefficients are given in Table 2. We parameterize the Mandel \& Agol (2002) model such that we fit for the orbital period $(P)$, time of transit $\left(T_{0}\right)$, impact parameter $(b)$, mean stellar density $\left(\rho_{\star}\right)$, scaled planet radius $\left(R_{p} / R_{\star}\right), e \cos \omega, e \sin \omega$ (where $e$ is eccentricity and $\omega$ is the periastron angle), and occultation (secondary eclipse) depth. 
Table 2

Planet Parameters Calculated from the MCMC Analysis of the Phase Curve of TrES-2b

\begin{tabular}{|c|c|c|c|}
\hline Parameter & Value & Literature Value $^{\mathrm{a}}$ & Reference \\
\hline Period (days) & $2.47061320_{-0.00000002}^{+0.00000002}$ & $2.47061892_{+0.00000012}^{+0.0000018}$ & Kipping \& Bakos (2011) \\
\hline$T_{0}(\mathrm{BJD})$ & $2454955.7625504_{-0.0000060}^{+0.0000051}$ & $2454849.526640_{-0.000021}^{+0.000022}$ & Kipping \& Bakos $(2011)^{\mathrm{b}}$ \\
\hline$R_{p} / R_{\star}$ & $0.125358_{-0.000024}^{+0.000019}$ & $0.1278_{-0.0018}^{+0.0029}$ & Kipping \& Bakos (2011) \\
\hline$b$ & $0.84293_{-0.00015}^{+0.00016}$ & $0.8418_{-0.0045}^{+0.0037}$ & Kipping \& Bakos (2011) \\
\hline Occultation depth (ppm) & $6.5_{-1.8}^{+1.7}$ & $16_{-14}^{+13}$ & Kipping \& Spiegel (2011) \\
\hline$e \sin \omega$ & $-0.00014_{-0.00036}^{+0.00012}$ & $-0.009_{-0.0029}^{+0.0024}$ & Kipping \& Bakos (2011) \\
\hline$e \cos \omega^{\mathrm{c}}$ & $-0.00016_{-0.00067}^{+0.00056}$ & $0.0005_{-0.0018}^{+0.0018}$ & Kipping \& Bakos (2011) \\
\hline$A_{e}(\mathrm{ppm})$ & $2.79_{-0.62}^{+0.44}$ & $1.50_{-0.93}^{+0.92}$ & Kipping \& Spiegel (2011) \\
\hline$A_{b}(\mathrm{ppm})$ & $3.44_{-0.33}^{+0.35}$ & $0.22_{-0.87}^{+0.88}$ & Kipping \& Spiegel (2011) \\
\hline$A_{r}(\mathrm{ppm})$ & $3.41_{-0.82}^{+0.55}$ & $6.5_{-1.9}^{+1.9}$ & Kipping \& Spiegel (2011) \\
\hline Flux offset (ppm) & $7.35_{-0.38}^{+0.38}$ & $\cdots$ & \\
\hline Phase lag ( $l$, radians) & $0.01_{-0.36}^{+0.37}$ & $\cdots$ & \\
\hline Limb-darkening coefficients $\left\{c_{1}, c_{2}, c_{3}, c_{4}\right\}$ & $\{0.4330,0.3552,0.0450,-0.1022\}$ & $\ldots$ & \\
\hline
\end{tabular}

Notes.

a Values taken from the literature are provided for comparison. Where the literature values are taken from Kipping \& Bakos (2011), we use their eccentric model.

b The transit epoch (T0) given by Kipping \& Bakos (2011) differs from ours owing to our fitting being free to choose any transit as the zero point.

c The uncertainties on $e \sin \omega$ have been inflated to account for Römer delay.

In addition, we simultaneously fit for the amplitude of the observable effects caused by ellipsoidal variations, Doppler beaming, and reflection/thermal emission from the planet using a modification to the method outlined in Faigler \& Mazeh (2011) as a function of orbital phase, $\phi$. The orbital phase is defined as

$$
\phi=\frac{\left(t-T_{0}\right)+P}{P}-\left\lfloor\frac{\left(t-T_{0}\right)+P}{P}\right\rfloor,
$$

where $P$ is the orbital period of the TrES-2b, $t$ is time, and $T_{0}$ is the time of the first transit. The expression $\lfloor x\rfloor \equiv$ floor $(x)$ where the "floor" function rounds down to the nearest integer. The phase $\phi$ runs from 0 to 1 and midtransit occurs at $\phi=0$. The change in brightness of the system as a function of phase can be described as

$$
\begin{gathered}
\frac{\Delta F}{F}=f-A_{e} \cos (4 \pi \phi-l)+A_{b} \sin 2 \pi \phi \\
-A_{r} \frac{\sin z+(\pi-z) \cos (z)}{\pi},
\end{gathered}
$$

where the amplitude coefficients $A_{e}, A_{b}$, and $A_{r}$ are owing to the effects of ellipsoidal variations, Doppler beaming, and reflection/thermal emission. $f$ is an arbitrary photometric zero point in flux which is needed because we do not precisely know the flux zero point before we fit a model. $l$ is a phase lag in the ellipsoidal variations where a significant detection would imply that the stellar tide is not exactly aligned with the planet. The amount by which the stellar tide lags behind the planetary orbit likely depends on the tidal quality factor of the star (Pfahl et al. 2008; Barnes 2010). $z$ is defined as

$$
\cos (z)=-\sin i \sin 2 \pi \phi,
$$

where $i$ is the inclination of the planet with respect to our line of sight with $90^{\circ}$ indicating the planet-star orbital plane is in our line of sight. $i$ is explicitly included in the description of the reflection but is included implicitly in the coefficients $A_{e}$ and $A_{b}$. For the reflection/thermal emission component in the

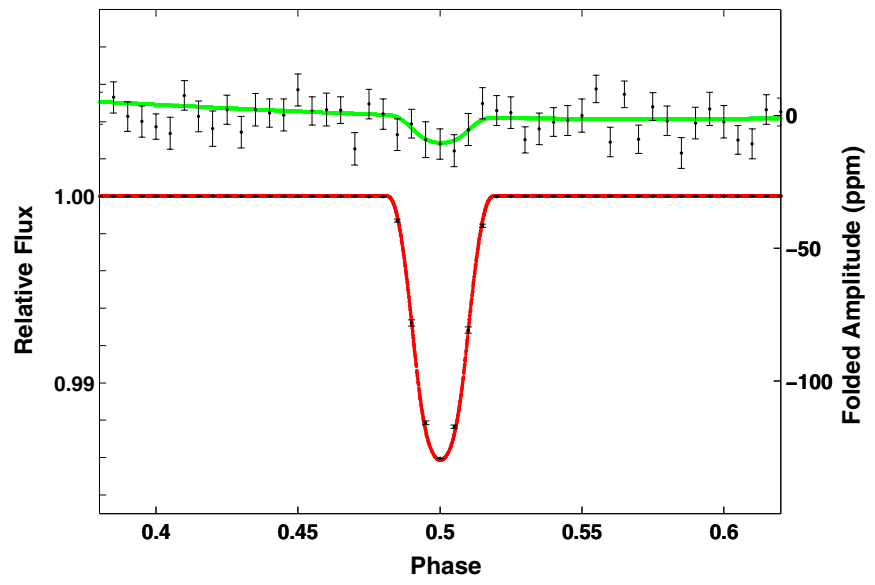

Figure 4. Folded and binned light curve of TrES-2 zoomed-in on the transit and the occultation. On top of this we plot the best-fitting photometric model-red for the transit and green for the occultation. The left-hand scale refers to the transit and the right-hand scale refers to the occultation.

(A color version of this figure is available in the online journal.)

above equation we have assumed a Lambertian phase function (Lambert 1760; Russell 1916; Sobolev 1975). The reason that $i$ must be explicitly included in the reflection/emission component is because the shape of the Lambertian phase function depends on the inclination of the planetary orbit whereas inclination affects only the amplitude of the ellipsoidal and Doppler beaming components.

A best-fit model was computed with a Levenberg-Marquardt algorithm (Moré 1978; Levenberg 1944; Marquardt 1963). We show the best-fitting model of the transit and occultation overplotted on the folded data in Figure 4. The best-fitting model parameters were then used to seed a Markov Chain Monte Carlo (MCMC) simulation (an introduction to transit model fitting using MCMC is given in Ford 2006 and Collier Cameron et al. 2007) with jumps defined using the Metropolis-Hastings algorithm. We adopted the asteroseismic derived value of $\rho_{\star}$ as a prior. We expect uncertainties on both the observed and 
modeled light curve data points to be normally distributed and therefore be roughly independent so we describe the likelihood for the model to match the observations as $e^{-\chi^{2} / 2}$, where $\chi^{2}$ is the standard $\chi^{2}$ statistic. The uncertainty on each data point $\sigma_{f, i}$ is taken directly from the Kepler data files. We calculated four chains of length $10^{5}$, each with perturbed starting parameters. The first $10 \%$ of chains were disregarded to avoid dependence on the initial starting position. All our parameter chains had a Gelman \& Rubin (1992) $\sqrt{\hat{R}}$ statistic of $<1$ which indicates good convergence, so we combined the four chains to calculate our final posterior distributions.

Our model does not include the effect a finite speed of light has on time of occultation relative to the time of transit. This effect is known as Römer delay and causes the occultation not to be seen at exactly phase 0.5 for a circular orbit. The Römer delay only affects the time of occultation and not the duration of the transit, and hence only the accuracy of $e \cos \omega$ is reduced (Sterne 1940; de Kort 1954). In order to account for this, we calculate the time delay we expect (Loeb 2005; Kaplan 2010) and inflate our uncertainty on $e \cos \omega$ by converting the time delay into $e \cos \omega$ (Winn 2011) and adding in quadrature. We note that the Römer delay owing to the motion of bodies in our solar system is explicitly corrected for.

We do not fit harmonics of the orbital period in order to describe an eccentric orbit (e.g., Mislis et al. 2012) because in initial transit fitting we found TrES-2b to be on an orbit very close to circular (the upper limit we derive for eccentricity would result in a $<1 \%$ change in the amplitude, which is below the uncertainty on any amplitude we measure). In Table 2, we report the median of our posterior distribution. The upper and lower bounds on the uncertainties are the 84.1 and 15.9 percentile of the marginalized posterior distribution, equal to the central $68.2 \%$ of the distribution. The best-fitting model of the out-oftransit/occultation effects (using the median of each chain) is shown in Figure 5.

We find significant detections of ellipsoidal, beaming, and reflection/thermal emission components in the phase curve. We do not detect any significant phase lag between the stellar tide raised by the planet and the orbital period of the planet. We also fit the three phase-curve components independently from the transit and occultation (phases around 0 and 0.5 were cut out), the results were consistent with the joint fit. Additionally, we tried including a $\sin (4 \pi \phi)$ component in the phase-curve fit as suggested by Faigler \& Mazeh (2011) and measured an amplitude consistent with zero. If the phase variations were due to noise there is no a priori reason why we would see positive amplitudes in the three physical components and a zero amplitude for the non-physical component. That the amplitude of a $\sin (4 \pi \phi)$ component is consistent with zero supports our conclusion that the phase-curve components are real.

In order to further increase our confidence that the phasecurve variations are not owing to random noise, we compared our model with a model containing no Doppler beaming, ellipsoidal variations, or reflection/emission by calculating the Deviance Information Criterion (DIC; Spiegelhalter et al. 2002) for each model. The difference in DIC values indicate that a model containing phase-curve variations is favored over the flat model by a factor of $>10^{38}$. A model containing ellipsoidal variations and Doppler beaming but no reflection is disfavored over a model including the reflection by a factor of $4.6 \times 10^{6}$. Likewise, models containing no Doppler beaming or no ellipsoidal variations but the other two effects are always strongly disfavored over a model with all three effects.

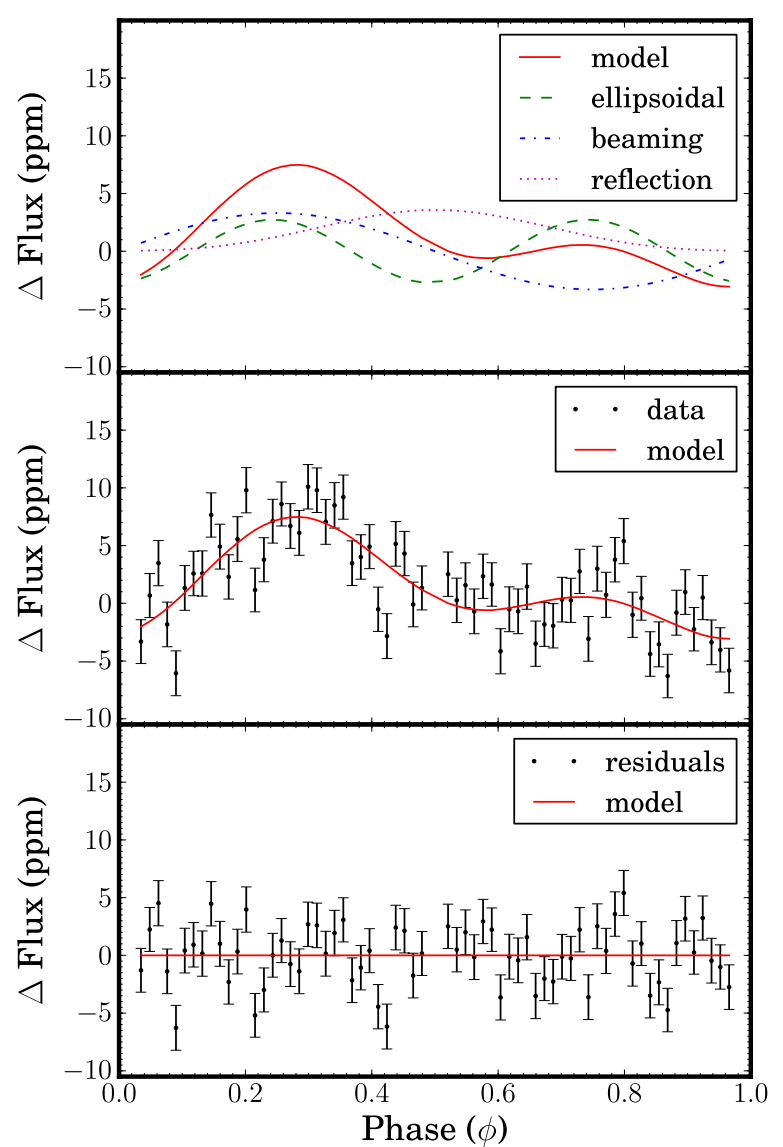

Figure 5. Model phase curve of TrES-2b is shown in the top panel with the three constituent parts which make up the model. The ellipsoidal effect (green dashed curve), the Doppler beaming (blue dot-dashed curve), and reflection/emission (purple dotted curve). The sum of these effects is the final model, shown by the red solid line in the top and the middle panel. The phase-folded Kepler data from Q0-11 is plotted in the central panel. The residuals of this fit are shown in the lower panel. The constant offset in our fits has been removed from the models shown for the ease of comparison and the transit and occultation has been cut out. Data have been binned in the figure with 15,000 short-cadence data points making up each bin, for the ease of interpretation. The fit described in the text was performed on un-folded and un-binned data.

(A color version of this figure is available in the online journal.)

An inclination change of $2 \times 10^{-4} \mathrm{deg} \mathrm{day}^{-1}$ has been reported by Mislis \& Schmitt (2009) and Mislis et al. (2010), suggesting a secular torque from an otherwise unseen companion. However, the detection was challenged from the ground by Scuderi et al. (2010), and the large magnitude was not confirmed by Schröter et al. (2012) on the basis of earlier Kepler data. We investigated this question again, now with a longer baseline.

We fixed $\rho_{\star}$ to its best-fitting value and allowed $i$ and $R_{p} / R_{\star}$ to solve for their best values for each quarter individually. The motivation for letting $R_{p} / R_{\star}$ float is not that we expect it to change physically, but that the quarterly changes in the observations may cause the dilution due to background stars to vary. Thus, we guard against misinterpreting a scale change as a duration change due to the rather V-shape light curve of this grazing planet.

The values of $i$ were scattered about the best fit, with no secular trend. We measure $d i / d t=(1.0 \pm 1.2) \times 10^{-6} \mathrm{deg}$ day $^{-1}$, i.e., no change to a $3 \sigma$ limit of $\sim 4.5 \times 10^{-6}$ deg day $^{-1}$. To interpret this limit in terms of a limit on perturbing planets, we simplify the expression from Ballard et al. (2010) using the approximation for the Laplace coefficient $b_{3 / 2}^{(1)}(\alpha) \rightarrow 3 \alpha$ for 
$\alpha \ll 1$, and find

$$
\begin{aligned}
M_{c}= & M_{\star} \frac{2}{3 \pi} \frac{1}{\Delta \Omega} \frac{P_{c}^{2}}{P_{b}} \frac{d i}{d t} \\
& <1.2 M_{\oplus}\left(\frac{P_{c}}{10 \text { days }}\right)^{2}\left(\frac{\Delta \Omega}{10^{\circ}}\right)^{-1},
\end{aligned}
$$

where $\Delta \Omega$ is the node of the perturbing planet on the sky plane, relative to the transiting planet, $M_{c}$ and $P_{c}$ are the period of the putative perturbing planet, and we have used our limit on $|d i / d t|$ in the final inequality. With this tight limit, we see that we are capable of detecting Earth-mass planets via their secular torques, as envisioned by Miralda-Escudé (2002), but see no evidence for their presence.

\section{PHYSICAL PARAMETERS FROM THE PHASE CURVE OF TrES-2B}

We use the three amplitudes calculated in our MCMC analysis to determine parameters that describe TrES-2b. For radial velocities much lower than the speed of light, the coefficient $A_{b}$ is described by

$$
A_{b}=\alpha_{b} \frac{K_{\mathrm{RV}}}{c},
$$

where $c$ is the speed of light and $\alpha_{b}$ is the photon-weighted bandpass-integrated beaming factor calculated in the manner described by Bloemen et al. (2011), where

$$
\alpha_{b}=\frac{\int \epsilon_{K} \lambda F_{\lambda} B d \lambda}{\int \epsilon_{K} \lambda F_{\lambda} d \lambda} .
$$

Here, $\epsilon_{K}$ is the Kepler response function, ${ }^{18} \lambda$ is the wavelength, and $B$ is the monochromatic beaming factor, which Loeb \& Gaudi (2003) give as

$$
B=5+\frac{d \ln F_{\lambda}}{d \ln \lambda}
$$

For TrES-2A, we compute $\alpha_{b}=3.97 \pm 0.03$ using ATLAS model spectra (Castelli \& Kurucz 2004) with parameters we derived in Section 2. $K_{\mathrm{RV}}$ is the radial velocity semi-amplitude which for a circular orbit is given by

$$
K_{\mathrm{RV}}=\left(\frac{2 \pi G}{P}\right)^{1 / 3} \frac{M_{p} \sin i}{M_{\star}^{2 / 3}} .
$$

Here, $G$ is the universal gravitational constant, $P$ is the orbital period of the planet, $M_{\star}$ is the mass of the star, and $M_{p}$ is the planetary mass. Therefore, if we measure $A_{b}$, we can find the mass ratio of the system.

We adopt the Faigler \& Mazeh (2011) parameterization of $A_{e}$, where

$$
A_{e}=\alpha_{e} \frac{M_{p}}{M_{\star}}\left(\frac{R_{\star}}{a}\right)^{3} \sin ^{2} i .
$$

We calculate $\alpha_{e}$ using a first-order approximation of the ellipsoidal variation given by Morris (1985) and Morris \& Naftilan (1993),

$$
\alpha_{e}=\frac{0.15(15+u)(1+g)}{3-u},
$$

\footnotetext{
18 The Kepler response function is available in the supplement to the Kepler Instrument Handbook.
}

where $u$ and $g$ are the linear limb-darkening and gravitydarkening parameters, respectively. We trilinearly interpolate the limb-and gravity-darkening coefficients calculated by Claret \& Bloemen (2011) from the grids in effective temperature, surface gravity, and metallicity, giving values of $u=0.580$ and $g=0.354$.

The geometric albedo is the ratio of the flux observed from a planet, compared to that of a perfect Lambert disk with the same radius as that of the planet. While in the solar system this observed flux is essentially entirely scattered star light, for hot Jupiters this planetary flux can be a mix of scattered light as well as thermal emission. Observationally, one cannot determine which is more important for a given planet, but with the aid of atmospheric models, the relative amounts of scattered and thermally emitted light can be estimated. We use the amplitude of the reflection/thermal emission component to calculate the geometric albedo, $A_{g}$, for TrES-2b in the Kepler bandpass using the equation

$$
A_{r}=A_{g}\left(\frac{R_{p}}{a}\right)^{2} .
$$

At each step in the Markov chains created in the previous section, we calculated the radial velocity semi-amplitude, planet mass, radius, density, and geometric albedo. The median values and uncertainties (central $68.27 \%$ of the distribution) on these parameters are given in Table 3. Using the Markov chains enables uncertainties and correlations between parameters to be propagated throughout this work.

\section{DISCUSSION}

Faigler \& Mazeh (2011) derive a parameter $\mathfrak{R}$ to denote the expected ratio of the ellipsoidal amplitude to the beaming amplitude which they define as

$$
\mathfrak{R} \equiv \frac{A_{e}}{A_{b}}=5 \frac{\alpha_{e}}{\alpha_{b} / 4} M_{\star}^{-4 / 3} R_{\star}^{3} P_{\text {orb }}^{-5 / 3} \sin i,
$$

where $M_{\star}$ and $R_{\star}$ are in units of solar mass and solar radius and $P_{\text {orb }}$ is in days. Note that we must divide our value of $\alpha_{b}$ by 4 in order to be consistent with Faigler \& Mazeh (2011). For TrES-2b, we find $\Re=0.81_{-0.13}^{+0.14}$, which is within $2 \sigma$ of the expected value of $1.34_{-0.17}^{+0.19}$. This difference is not particularly significant and we do not find this overly concerning.

We measure a radius for TrES-2b of $1.162_{-0.024}^{+0.020} R_{\text {Jup }}$ which is consistent with previously published values (O'Donovan et al. 2006; Sozzetti et al. 2007; Daemgen et al. 2009; Southworth 2011). However, the radial velocity semi-amplitude we calculate from photometry $\left(249_{-28}^{+24} \mathrm{~m} \mathrm{~s}^{-1}\right)$ is significantly larger than the value measured by O'Donovan et al. (2006) of $181.3 \pm 2.6 \mathrm{~m} \mathrm{~s}^{-1}$ using spectroscopic radial velocity data, hence the mass derived from Doppler beaming is also on the high side. Our derived mass is $\sim 1 \sigma$ higher than the mass found by O'Donovan et al. (2006) and $2 \sigma$ higher than the mass found by Sozzetti et al. (2007) who use the same radial velocity data as O'Donovan et al. but with revised stellar parameters.

Our masses derived from the ellipsoidal variations and from Doppler beaming differ by $2 \sigma$. The mass derived from beaming is usually taken to be the ground truth because it can be calculated in a relatively model-independent way whereas Equation (11) is an approximation and relies on the accuracy of the equations used to describe limb and gravity darkening. Shporer et al. (2011) discuss the limitations of this approximation for an earlier-type star such as KOI-13A, however, in 
Table 3

Derived Parameters of TrES-2b

\begin{tabular}{|c|c|c|c|}
\hline Parameter & Value & Literature Value & Reference \\
\hline Radial velocity semi-amplitude $\left(\mathrm{m} \mathrm{s}^{-1}\right)$ & $249_{-28}^{+24}$ & $181.3 \pm 2.6$ & O’Donovan et al. (2006) \\
\hline Planet mass from beaming $\left(M_{\mathrm{Jup}}\right)$ & $1.61_{-0.18}^{+0.17}$ & $\cdots$ & \\
\hline Planet mass from ellipsoidal variations $\left(M_{\mathrm{Jup}}\right)$ & $1.06_{-0.23}^{+0.28}$ & $\cdots$ & \\
\hline Weighted average planet mass $\left(M_{\text {Jup }}\right)$ & $1.44 \pm 0.21$ & $1.206 \pm 0.045$ & Southworth (2011) \\
\hline Planet density $\left(\mathrm{g} \mathrm{cm}^{-3}\right)$ & $1.14_{-0.17}^{+0.12}$ & $\mathrm{a}$ & \\
\hline Planet radius $\left(R_{\mathrm{Jup}}\right)$ & $1.162_{-0.024}^{+0.020}$ & $1.187_{-0.035}^{+0.034}$ & Kipping \& Bakos (2011) \\
\hline$a / R_{\star}$ & $7.931_{-0.046}^{+0.056}$ & $8.06_{-0.021}^{+0.025}$ & Kipping \& Bakos (2011) \\
\hline Semimajor axis (AU) & $0.03503_{-0.00073}^{+0.00053}$ & $0.03563_{-0.000058}^{+0.00048}$ & Kipping \& Bakos (2011) \\
\hline Inclination $\left(^{\circ}\right)$ & $83.881_{-0.043}^{+0.038}$ & $84.07_{-0.31}^{+0.34}$ & Kipping \& Bakos (2011) \\
\hline Geometric albedo $A_{g}$ & $0.0136_{-0.0033}^{+0.0022}$ & $0.0253 \pm 0.0072$ & Kipping \& Spiegel (2011) \\
\hline
\end{tabular}

Notes.

The table is split between those values which are related to the photometric determination of the planet mass but we consider less precise than the literature values obtained from radial velocity observations and values obtained from the transit model which exceed the precision found in the literature. We recommend parameters derived from the transit model be used in future studies of TrES-2.

${ }^{\text {a }}$ We do not give a density from the literature here owing to the two different sources from which we take mass and radius.

the case of a star with a convective envelope such as TrES2A the approximation should describe the amplitude owing to ellipsoidal variations reasonably well. For the planet mass we adopt the weighted average of the mass derived from Doppler beaming and from ellipsoidal variations where the weighting is based on the standard deviation the MCMC chain masses as a proxy for uncertainty. This results in the mass of TrES-2b being $1.44 \pm 0.21 M_{\text {Jup }}$. We stress that this value does not supersede previous measurements from spectroscopic radial velocity observations, but merely demonstrates that this technique can be used to determine a planet mass consistent with other observations albeit at lower accuracy. However, the ability to derive masses consistent with valuable multiple epoch, high-resolution spectra for radial velocities should not be overlooked.

As discussed earlier, Römer delay is not included in our model. We estimate that the observed time of occultation will be offset by 34.5 s compared to what would be seen if the speed of light was infinite (Kaplan 2010; Bloemen et al. 2012). The Römer delay equates to a change in $e \cos \omega$ of 0.00051 (Winn 2011). We add this value in quadrature to our previously derived uncertainties on $e \cos \omega$ to give a final value of $-0.00016_{-0.00067}^{+0.00056}$. This equates to an eccentricity of $0.0002_{-0.0002}^{+0.0010}$. This tight constraint on the eccentricity of the planet (the $3 \sigma$ upper limit is 0.0025 ) is consistent with the expectations of tidal dissipation. This low eccentricity suggests that the method of Ragozzine \& Wolf (2009) for observing apsidal precession due to the planetary interior is not possible with the existing data.

A growing number of planets have infrared emission spectroscopy from Spitzer and adding the Kepler data point to the existing data can be an important extra constraint for differentiating inverted and non-inverted atmospheres (Christiansen et al. 2010). We have modeled the atmosphere of TrES-2b using the methods described in Fortney \& Marley (2007) and Fortney et al. (2008). In Figure 6, we compare the dayside planet-tostar flux ratio data to the three atmosphere models. The models shown use a redistribution factor $f$ of 0.5 or 0.45 to simulate redistribution of absorbed stellar flux over the entire day side ( $f=0.5$ ) or with a slight loss to the night side $f=0.45$. The yellow model does not include $\mathrm{TiO} / \mathrm{VO}$ as gaseous absorbers, leaving gaseous $\mathrm{Na}$ and $\mathrm{K}$ to absorb optical flux at much higher pressures, and a temperature inversion does not occur. The blue and red models include equilibrium mixing ratios of $\mathrm{TiO} / \mathrm{VO}$ and possess temperature inversions. TiO and $\mathrm{VO}$ gases have exceptionally strong optical opacity, and can lead to temperature inversions (Hubeny et al. 2003; Fortney et al. 2006).

The Spitzer data plotted in Figure 6 are from O'Donovan et al. (2010) and the $K_{\mathrm{S}}$ datum from Croll et al. (2010). Croll et al. used similar methods to Fortney et al. to find a best-fit atmosphere model that included a temperature inversion, but it was only a marginally better fit than the no inversion model. However, with the inclusion of the Kepler data point, a model with a temperature inversion is now more strongly preferred. Spiegel \& Burrows (2010) have also modeled TrES-2b, using the same data shown here, except the Kepler data point was a preliminary number (Kipping \& Bakos 2011). These authors find a significantly better fit with a temperature inversion as well. Models that lack $\mathrm{TiO}$ or a similar high-altitude strong optical absorber are often too bright at optical wavelengths. This can be seen in Figure 6 and Spiegel \& Burrows (2010, Figure 2(a)). The very low geometric albedo detected for TrES$2 \mathrm{~b}$ of $0.0136_{-0.0033}^{+0.0022}$ is in line with model predictions for cloudfree hot Jupiter atmospheres (Sudarsky et al. 2000).

The relatively small day-to-night brightness contrast of $3.41_{-0.82}^{+0.55} \mathrm{ppm}$ is quite interesting, but potentially difficult to understand in a simple way. If $\mathrm{TiO}$ (or a similar optical absorber) is present on the day side, it may well condense out on the night side (e.g., Showman et al. 2009). This could mean the depth probed in the Kepler bandpass could be at a pressure $\sim 100 \times$ larger on the night side. This same dramatic change in opacity likely does not happen in the near- or mid-infrared (Knutson et al. 2009), since $\mathrm{H}_{2} \mathrm{O}$ is the dominant absorber in both hemispheres, and the day and night abundances of $\mathrm{CO}$ and $\mathrm{CH}_{4}$ may be homogenized due to vertical and horizontal mixing (Cooper \& Showman 2006).

A strong possibility for TrES-2b is that the brightness temperature of the inverted dayside atmosphere probes pressures of $\sim 10$ mbar, which may be quite similar in temperature to that on the night side near 1 bar. Another possibility for the warm night side of TrES-2b could be energy dissipation due to a variety of potential mechanisms (Showman \& Guillot 2002; Arras \& Socrates 2010; Batygin \& Stevenson 2010; Youdin \& Mitchell 2010), which could be expressed as a higher temperature at 1 bar. 


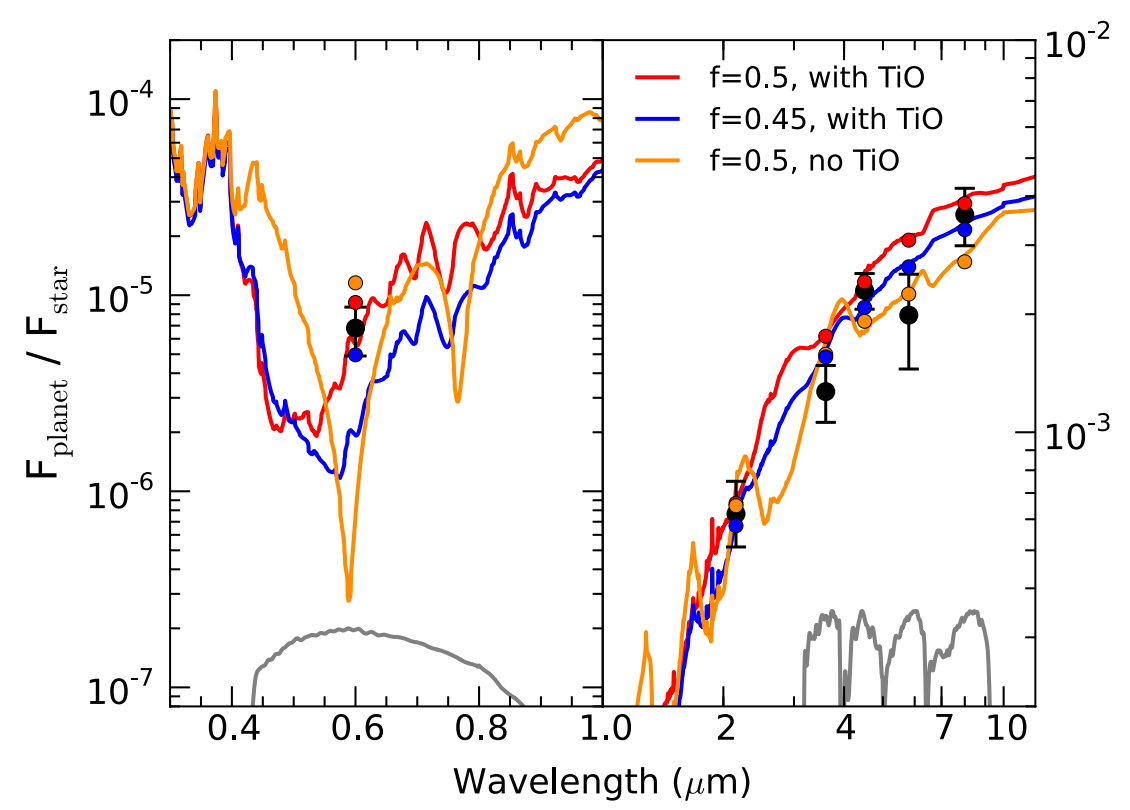

Figure 6. Left panel: planet-to-star flux ratios at optical wavelengths. The transmission function of the Kepler bandpass is shown, as well as the measured occultation depth (black, with error bars). Right panels: planet-to-star flux ratios at infrared wavelengths. The transmission function of the Spitzer IRAC bandpasses is shown, as well as the measured occultation depths (black, with error bars) from O'Donovan et al. (2010) at IRAC wavelengths and Croll et al. (2010) at $K_{\mathrm{s}}$. The best-fit model is shown in blue, which features a dayside temperature inversion, due to TiO gas, and modest redistribution of absorbed flux to the night side. When fitting models, we convolve the bandpass of the observations with the models.

(A color version of this figure is available in the online journal.)

We note that Welsh et al. (2010) also found a small $(\sim 300 \mathrm{~K})$ day-night brightness temperature contrast for HAT-P-7b in the Kepler band, although Jackson et al. (2012), with more data, suggest the difference is greater than $700 \mathrm{~K}$.

The dayside to nightside planet flux ratio we determine is lower than that found by Kipping \& Spiegel (2011) who detected a phase-curve reflection/emission component with a full amplitude of $6.5 \pm 1.9 \mathrm{ppm}$ which is higher than the $3.41_{-0.82}^{+0.55} \mathrm{ppm}$ we detect, although still within $1 \sigma-2 \sigma$.

\section{SUMMARY}

We have discovered ellipsoidal variations and Doppler beaming in the phase curve of the TrES-2 star-planet system. An asteroseismic analysis of the solar-like oscillations on the star allows us to derive an accurate stellar mass and radius. Using the approach of Faigler \& Mazeh (2011), we derived a mass of TrES-2b of $1.61_{-0.18}^{+0.16} M_{\text {Jup }}$ from the Doppler beaming effect and $1.06_{-0.23}^{+0.28} M_{\text {Jup }}$ from the ellipsoidal variations. That these two values are independent and that they agree to within $2 \sigma$ and are within $1 \sigma-3 \sigma$ of literature values strengthens our belief that the variations are not instrumental or induced by stellar variability. We are further convinced that what we detect is truly induced by the planet because the ratio of ellipsoidal to beaming amplitudes agrees within $\sim 2 \sigma$ of theoretically expected values.

We detect a difference between the dayside and the nightside flux from TrES-2b of the level of $3.41_{-0.82}^{+0.55} \mathrm{ppm}$, lower than the amplitude reported by Kipping \& Spiegel (2011). This suggests that TrES-2b, the darkest known exoplanet, is even darker than previously thought.

This paper includes data collected by the Kepler mission. Funding for the Kepler mission is provided by the NASA Science Mission directorate. All Kepler data presented in this paper were obtained from the Mikulski Archive for Space Telescopes (MAST) at the Space Telescope Science Institute
(STScI). STScI is operated by the Association of Universities for Research in Astronomy, Inc., under NASA contract NAS526555. Support for MAST for non-HST data is provided by the NASA Office of Space Science via grant NNX09AF08G and by other grants and contracts. S.B. acknowledges funding from the European Research Council under the European Community's Seventh Framework Programme (FP7/2007-2013)/ERC grant agreement No. 227224 (PROSPERITY), as well as from the Research Council of KU Leuven grant agreement GOA/2008/04. We thank Simchon Faigler and the Kepler Science Team for providing insightful comments during the preparation of this manuscript. D.H. is supported by appointment to the NASA Postdoctoral Program at Ames Research Center, administered by Oak Ridge Associated Universities through a contract with NASA.

\section{REFERENCES}

Alonso, R., Brown, T. M., Torres, G., et al. 2004, ApJ, 613, L153

Ammler-von Eiff, M., Santos, N. C., Sousa, S. G., et al. 2009, A\&A, 507, 523 Arras, P., \& Socrates, A. 2010, ApJ, 714, 1

Bakos, G. Á., Lázár, J., Papp, I., Sári, P., \& Green, E. M. 2002, PASP, 114, 974 Ballard, S., Christiansen, J. L., Charbonneau, D., et al. 2010, ApJ, 716, 1047 Barclay, T., Still, M., Jenkins, J. M., Howell, S. B., \& Roettenbacher, R. M. 2012, MNRAS, 422, 1219

Barnes, R. (ed.) 2010, Formation and Evolution of Exoplanets (Weinheim: Wiley-VCH)

Basu, S., Chaplin, W. J., \& Elsworth, Y. 2010, ApJ, 710, 1596

Basu, S., Verner, G. A., Chaplin, W. J., \& Elsworth, Y. 2012, ApJ, 746, 76

Batygin, K., \& Stevenson, D. J. 2010, ApJ, 714, L238

Bloemen, S., Marsh, T. R., Degroote, P., et al. 2012, MNRAS, 422, 2600

Bloemen, S., Marsh, T. R., Østensen, R. H., et al. 2011, MNRAS, 410, 1787

Borucki, W. J., Koch, D., Basri, G., et al. 2010, Science, 327, 977

Carter, J. A., Agol, E., Chaplin, W. J., et al. 2012, Science, 337, 556

Castelli, F., \& Kurucz, R. L. 2004, arXiv:astro-ph/0405087

Chaplin, W. J., Kjeldsen, H., Christensen-Dalsgaard, J., et al. 2011, Science, 332,213

Christensen-Dalsgaard, J. 1988, in IAU Symp. 123, Advances in Helio- and Asteroseismology, ed. J. Christensen-Dalsgaard \& S. Frandsen (Cambridge: Cambridge Univ. Press), 295 
Christensen-Dalsgaard, J., Kjeldsen, H., Brown, T. M., et al. 2010, ApJ, 713, L164

Christiansen, J. L., Ballard, S., Charbonneau, D., et al. 2010, ApJ, 710, 97

Christiansen, J. L., Van Cleve, J. E., Jenkins, J. M., et al. 2012, Kepler Data Characteristics Handbook, KSCI-19040-003 (Moffett Field, CA: NASA Ames Research Center)

Claret, A., \& Bloemen, S. 2011, A\&A, 529, A75

Collier Cameron, A., Wilson, D. M., West, R. G., et al. 2007, MNRAS, 380,1230

Cooper, C. S., \& Showman, A. P. 2006, ApJ, 649, 1048

Coughlin, J. L., \& López-Morales, M. 2012, ApJ, 750, 100

Croll, B., Albert, L., Lafreniere, D., Jayawardhana, R., \& Fortney, J. J. 2010, ApJ, 717,1084

Daemgen, S., Hormuth, F., Brandner, W., et al. 2009, A\&A, 498, 567

de Kort, J. J. M. A. 1954, Ric. Astron., 3, 109

Demory, B.-O., Seager, S., Madhusudhan, N., et al. 2011, ApJ, 735, L12

Désert, J.-M., Charbonneau, D., Demory, B.-O., et al. 2011a, ApJS, 197, 14

Désert, J.-M., Charbonneau, D., Fortney, J. J., et al. 2011b, ApJS, 197, 11

Fabrycky, D. C., Ford, E. B., Steffen, J. H., et al. 2012, ApJ, 750, 114

Faigler, S., \& Mazeh, T. 2011, MNRAS, 415, 3921

Ford, E. B. 2006, ApJ, 642, 505

Ford, E. B., Fabrycky, D. C., Steffen, J. H., et al. 2012a, ApJ, 750, 113

Ford, E. B., Ragozzine, D., Rowe, J. F., et al. 2012b, ApJ, 756, 185

Fortney, J. J., Demory, B.-O., Désert, J.-M., et al. 2011, ApJS, 197, 9

Fortney, J. J., Lodders, K., Marley, M. S., \& Freedman, R. S. 2008, ApJ, 678,1419

Fortney, J. J., \& Marley, M. S. 2007, ApJ, 666, L45

Fortney, J. J., Saumon, D., Marley, M. S., Lodders, K., \& Freedman, R. S. 2006, ApJ, 642, 495

Gelman, A., \& Rubin, D. 1992, Stat. Sci., 7, 457

Gilliland, R. L., Jenkins, J. M., Borucki, W. J., et al. 2010, ApJ, 713, L160

Harrington, J., Hansen, B. M., Luszcz, S. H., et al. 2006, Science, 314, 623

Hekker, S., Elsworth, Y., De Ridder, J., et al. 2011, A\&A, 525, A131

Hills, J. G., \& Dale, T. M. 1974, A\&A, 30, 135

Holman, M. J., Winn, J. N., Latham, D. W., et al. 2007, ApJ, 664, 1185

Howell, S. B., Rowe, J. F., Bryson, S. T., et al. 2012, ApJ, 746, 123

Hubeny, I., Burrows, A., \& Sudarsky, D. 2003, ApJ, 594, 1011

Huber, D., Bedding, T. R., Stello, D., et al. 2011, ApJ, 743, 143

Huber, D., Ireland, M. J., Bedding, T. R., et al. 2012, ApJ, 760, 32

Huber, D., Stello, D., Bedding, T. R., et al. 2009, Commun. Asteroseismol., 160,74

Husnoo, N., Pont, F., Mazeh, T., et al. 2012, MNRAS, 422, 3151

Jackson, B. K., Lewis, N. K., Barnes, J. W., et al. 2012, ApJ, 751, 112

Kallinger, T., Mosser, B., Hekker, S., et al. 2010, A\&A, 522, A1

Kaplan, D. L. 2010, ApJ, 717, L108

Kinemuchi, K., Barclay, T., Fanelli, M., et al. 2012, PASP, 124, 963

Kipping, D., \& Bakos, G. 2011, ApJ, 733, 36

Kipping, D. M., \& Spiegel, D. S. 2011, MNRAS, 417, L88

Kjeldsen, H., Bedding, T. R., Arentoft, T., et al. 2008, ApJ, 682, 1370

Knutson, H. A., Charbonneau, D., Cowan, N. B., et al. 2009, ApJ, 690, 822

Koch, D. G., Borucki, W. J., Basri, G., et al. 2010, ApJ, 713, L79

Lambert, J. H. 1760, Photometria sive de mensure de gratibus luminis, colorum umbrae (Augsburg: Eberhard Klett)

Levenberg, K. 1944, Q. Appl. Math., 2, 164

Lissauer, J. J., Fabrycky, D. C., Ford, E. B., et al. 2011, Nature, 470, 53

Loeb, A. 2005, ApJ, 623, L45

Loeb, A., \& Gaudi, B. S. 2003, ApJ, 588, L117

Mandel, K., \& Agol, E. 2002, ApJ, 580, L171

Marquardt, D. W. 1963, J. Soc. Ind. Appl. Math., 11, 431

Maxted, P. F. L., Marsh, T. R., \& Moran, C. K. J. 2000, MNRAS, 319, 305

Mayor, M., Udry, S., Lovis, C., et al. 2009, A\&A, 493, 639

Mazeh, T. 2008, in Tidal Effects in Stars, Planets, \& Disks, ed. M.-J. Goupil \& J.-P. Zahn (EAS Pub. Series 29; Les Ulis: EDP), 1

Mazeh, T., \& Faigler, S. 2010, A\&A, 521, L59

Mazeh, T., Nachmani, G., Sokol, G., Faigler, S., \& Zucker, S. 2012, A\&A, 541, A56
McCullough, P. R., Stys, J. E., Valenti, J. A., et al. 2005, PASP, 117, 783

Miglio, A. 2012, in Red Giants as Probes of the Structure and Evolution of the Milky Way, Astrophysics and Space Science Proceedings, ed. A. Miglio, J. Montalban, \& A. Noels (Springer-Verlag: Berlin-Heidelberg), 11

Miralda-Escudé, J. 2002, ApJ, 564, 1019

Mislis, D., Heller, R., Schmitt, J. H. M. M., \& Hodgkin, S. 2012, A\&A, 538, A4

Mislis, D., \& Hodgkin, S. 2012, MNRAS, 422, 1512

Mislis, D., \& Schmitt, J. H. M. M. 2009, A\&A, 500, L45

Mislis, D., Schröter, S., Schmitt, J. H. M. M., Cordes, O., \& Reif, K. 2010, A\&A, 510, A107

Moré, J. 1978, in Numerical Analysis, Vol. 630, ed. G. Watson (Berlin: Springer), 105

Morris, S. L. 1985, ApJ, 295, 143

Morris, S. L., \& Naftilan, S. A. 1993, ApJ, 419, 344

O’Donovan, F. T., Charbonneau, D., Harrington, J., et al. 2010, ApJ, 710,1551

O’Donovan, F. T., Charbonneau, D., Mandushev, G., et al. 2006, ApJ, 651, L61

Pfahl, E., Arras, P., \& Paxton, B. 2008, ApJ, 679, 783

Pietrinferni, A., Cassisi, S., Salaris, M., \& Castelli, F. 2004, ApJ, 612, 168

Pollacco, D. L., Skillen, I., Collier Cameron, A., et al. 2006, PASP, 118, 1407

Quintana, E. V., Rowe, J. F., Barclay, T., et al. 2012, ApJ, submitted

Ragozzine, D., \& Wolf, A. S. 2009, ApJ, 698, 1778

Rowe, J. F., Matthews, J. M., Seager, S., et al. 2006, ApJ, 646, 1241

Rowe, J. F., Matthews, J. M., Seager, S., et al. 2008, ApJ, 689, 1345

Russell, H. N. 1916, ApJ, 43, 173

Schneider, J., Dedieu, C., Le Sidaner, P., Savalle, R., \& Zolotukhin, I. 2011, A\&A, 532, A79

Schröter, S., Schmitt, J. H. M. M., \& Müller, H. M. 2012, A\&A, 539, A97

Scuderi, L. J., Dittmann, J. A., Males, J. R., Green, E. M., \& Close, L. M. 2010, ApJ, 714, 462

Showman, A. P., Fortney, J. J., Lian, Y., et al. 2009, ApJ, 699, 564

Showman, A. P., \& Guillot, T. 2002, A\&A, 385, 166

Shporer, A., Jenkins, J. M., Rowe, J. F., et al. 2011, AJ, 142, 195

Silva Aguirre, V., Casagrande, L., Basu, S., et al. 2012, ApJ, 757, 99

Smith, J. C., Stumpe, M. C., Van Cleve, J. E., et al. 2012, PASP, 124, 1000

Snellen, I. A. G., de Mooij, E. J. W., \& Albrecht, S. 2009, Nature, 459, 543

Sobolev, V. V. 1975, Light Scattering in Planetary Atmospheres (Oxford: Pergamon Press), 263

Southworth, J. 2009, MNRAS, 394, 272

Southworth, J. 2011, MNRAS, 417, 2166

Sozzetti, A., Torres, G., Charbonneau, D., et al. 2007, ApJ, 664, 1190

Spiegel, D. S., \& Burrows, A. 2010, ApJ, 722, 871

Spiegelhalter, D. J., Best, N. G., Carlin, B. P., \& Van Der Linde, A. 2002, J. R. Stat. Soc., Series B (Stat. Methodol.), 64, 583

Steffen, J. H., Fabrycky, D. C., Ford, E. B., et al. 2012a, MNRAS, 421, 2342

Steffen, J. H., Ragozzine, D., Fabrycky, D. C., et al. 2012b, Proc. Natl Acad. Sci., 109, 7982

Stello, D., Chaplin, W. J., Basu, S., Elsworth, Y., \& Bedding, T. R. 2009a, MNRAS, 400, L80

Stello, D., Chaplin, W. J., Bruntt, H., et al. 2009b, ApJ, 700, 1589

Sterne, T. E. 1940, Proc. Natl Acad. Sci., 26, 36

Stumpe, M. C., Smith, J. C., Van Cleve, J. E., et al. 2012, PASP, 124, 985

Sudarsky, D., Burrows, A., \& Pinto, P. 2000, ApJ, 538, 885

Torres, G., Fischer, D. A., Sozzetti, A., et al. 2012, ApJ, 757, 161

Ulrich, R. K. 1986, ApJ, 306, L37

Verner, G. A., Elsworth, Y., Chaplin, W. J., et al. 2011, MNRAS, 415, 3539

Vogt, S. S., Marcy, G. W., Butler, R. P., \& Apps, K. 2000, ApJ, 536, 902

Welsh, W. F., Orosz, J. A., Seager, S., et al. 2010, ApJ, 713, L145

White, T. R., Bedding, T. R., Stello, D., et al. 2011, ApJ, 743, 161

Winn, J. N. 2011, in Exoplanets, ed. S. Seager (Tucson, AZ: Univ. Arizona Press), 55

Wright, J. T., Fakhouri, O., Marcy, G. W., et al. 2011, PASP, 123, 412

Youdin, A. N., \& Mitchell, J. L. 2010, ApJ, 721, 1113

Zucker, S., Mazeh, T., \& Alexander, T. 2007, ApJ, 670, 1326 